\title{
Catalog of Long-Term Transient Sources in the First 10 Years of Fermi-LAT Data
}

\section{Isabella Mereu ${ }^{a, *}$, Sara Cutini ${ }^{a}$, Elisabetta Cavazzuti ${ }^{b}$ and Gino Tosti ${ }^{a, c}$ on behalf of the Fermi-LAT Collaboration}

${ }^{a}$ Istituto Nazionale di Fisica Nucleare, Sezione di Perugia,I-06123 Perugia, Italy

${ }^{b}$ Italian Space Agency, Via del Politecnico snc, 00133 Roma, Italy

${ }^{c}$ Dipartimento di Fisica, Università degli Studi di Perugia,I-06123 Perugia, Italy E-mail: mereuisabella@gmail.comn, sara.cutini@pg.infn.it, elisabetta.cavazzuti@asi.it, gino.tosti@unipg.it

The catalog of long-term transient sources comprises sources that were detected on monthly time intervals during the first decade of Fermi-LAT operations (1FLT). The monthly time scale allows us to identify transient and variable sources that may have not been reported in Fermi-LAT general catalogs. The analysis was performed for photon energies between 0.1 and $300 \mathrm{GeV}$ using the Pass-8 event-level selection. In the analysis we considered only photons with $|\mathrm{b}|>10^{\circ}$ to exclude the Galactic plane and therefore to avoid confusion with low-latitude diffuse emission. We have analyzed 120 months and also performed a 15-day shift of each month in order to not lose any flare at the edges of each time bin. The monthly datasets were analyzed using a wavelet-based source detection algorithm that provided the candidate new transient sources. The transient candidates were then analyzed using the standard Fermi-LAT maximum likelihood analysis method. The resulting catalog list has 142 different sources not included in 4FGL-DR2 and detected with a statistical significance above $4 \sigma$ in at least one monthly bin. About $70 \%$ are associated with spectrally soft AGN-type counterparts, principally blazar candidates of uncertain type and flatspectrum radio quasars, and about $30 \%$ of 1 FLT sources remain unassociated. This is similar to the fraction of unassociated sources found in the Fermi-LAT general catalogs. The median $\gamma$-ray spectral index of the 1FLT-AGN sources is softer than the median index reported in the latest Fermi-LAT AGN general catalog (4LAC). In the 1FLT catalog are reported 6 sources listed also in a previous general catalog (1-3FGL).

$37^{\text {th }}$ International Cosmic Ray Conference (ICRC 2021)

July 12th - 23rd, 2021

Online - Berlin, Germany

\footnotetext{
${ }^{*}$ Presenter
} 


\section{Introduction}

The Large Area Telescope (LAT) on board the Fermi Gamma-ray Space Telescope is an imaging pair-conversion detector operating in the energy range from $20 \mathrm{MeV}$ to $>300 \mathrm{GeV}$. It has a field of view of $2.7 \mathrm{sr}$ at $1 \mathrm{GeV}$ and can provide an image of the entire sky approximately every three hours [1]. This, together with its large collecting area, makes the LAT well suited to the investigation of $\gamma$-ray variable and transient sources. In the Fermi-LAT Fourth Source Catalog Data Release 2 (hereafter 4FGL-DR2; [2]), based on 10 years of data, the fraction of sources associated with flat-spectrum radio quasars (FSRQs) is less than that associated with BL Lacs. This difference in populations was also found in the previous XFGL catalogs, with the fraction of new sources slightly higher for hard-spectrum sources than for soft-spectrum ones relative to the previous catalog. This could be related to the different variability properties of FSRQs and BL Lacs as well as to the typically soft $\gamma$-ray spectra of FSRQs, while BL Lacs generally show harder spectra [3]. In fact the sensitivity of the Fermi-LAT improves at a faster rate as a function of exposure for harder sources.

Following these premises, soft-spectrum objects that have low duty cycle would not be detectable in the years-long integration of xFGL catalogs but could be detectable during brief periods of enhanced $\gamma$-ray activity.

\section{The 1FLT Catalog}

The data used in this work encompass a 10-year period starting from 2008 August 4 15:43:36 UTC. They are analyzed in monthly time bins, where one time bin (TBIN) is defined as $2.63 \mathrm{Ms}(\sim$ 30 days). To improves the sensitivity for sources that may display variable activity at the edges of the time bins, we repeated the monthly binned analysis selecting data with a 15-day shift. The 1FLT is constructed in the energy range from $100 \mathrm{MeV}$ to $300 \mathrm{GeV}$ using the Pass 8 event reconstruction. The exposure of the Fermi-LAT is fairly uniform across the sky, but the brightness of the interstellar diffuse $\gamma$-ray background, and hence the sensitivity for source detection, depends strongly on the Galactic latitude. For this reason consider only data with Galactic latitude $|b|>10^{\circ}$. Adopting this procedure we have analyzed a total of two sets of 120 monthly data sets. In the following we refer to these two sets as follows: "nominal" for the first set starting on 2008 August 4, and "shifted" for the second set starting on 2008 August 19.

The sky of each of the 240 monthly TBINs is divided into 192 circular regions of interest (ROIs). The detection of the candidate sources (seeds) in each ROI is performed using a wavelet transform analysis [4] using the PGWave tool [5]. After the seed extraction we performed a binned Maximum Likelihood (ML) analysis on all seeds that had an angular distance greater than 50 arcmin from any 4FGL-DR2 source. For each point source we assumed a power-law spectrum defined as $d N / d E=K *\left(E / E_{0}\right)^{-\Gamma}$, where $K$ is the flux scale factor, $\Gamma$ the spectral index, and $E_{0}$ is the reference energy $\left(E_{0}=1 \mathrm{GeV}\right)$. The ROI model used in the ML analysis contained all PGWave seeds found (including also the ones associated with 4FGL-DR2 sources) in the selected month in a ROI of $10^{\circ} \times 10^{\circ}$ square, centered at the position of the target, along with the Galactic and isotropic diffuse backgrounds ${ }^{1}$. To perform the analysis we used the Fermitools 1.2.1 package (FSSC) ${ }^{2}$ and

${ }^{1}$ https://fermi.gsfc.nasa.gov/ssc/data/access/lat/BackgroundModels.html

2https://fermi.gsfc.nasa.gov/ssc/data/analysis/ 
the Fermipy 0.18.0 software package [6]. The ML analysis provides the evaluation of the Test Statistic, TS, defined as TS $=2 \Delta \log \mathcal{L}$ [7] which quantifies how significantly a source emerges from the background comparing the likelihood function $\mathcal{L}$ with and without a source. We use TS $=25$ as the detection threshold, corresponding to a significance of $\sim 4 \sigma$ assuming a $\chi^{2}$ distribution with 4 degrees of freedom (position and spectral parameters of a power-law source.

\subsection{False positive estimation and source detection efficiency}

Over month-long time scales the false positives are dominated by statistical fluctuations of the background rather than systematic effects. Therefore we simulated 120 month-long data sets, including in the model only the Galactic and the extragalactic diffuse emissions and we applied to the simulations the same analysis of the 1FLT. In the 120 simulated skies we found 12 spurious detections with TS $>25$ over $\sim 24000$ seeds, and 1 detection with TS $>30$. In the estimation of the spurious detections in the real data these numbers should be multiplied by a factor of two to account also for the shifted months. Therefore we may expect 24 fake detections with TS $>25$. Since our adopted threshold of TS $>25$ still includes a non-negligible number of spurious sources, in the 1FLT catalog TS $<30$ candidate sources are flagged as low-confidence when they are detected in only one TBIN. Each individual low-confidence source has a probability of about $34 \%$ of being spurious.

To assess the detection efficiency we used 4FGL high latitude pulsars (PSRs), astrophysical $\gamma$-ray sources known to be steady in flux. The majority of sources taken in consideration for the estimation of efficiency are millisecond pulsars. For each TBIN of data we applied the analysis procedures and we compared the number of detections obtained as a function of energy flux to the total number of 4FGL pulsars. As suggested in [8], we modeled the detection efficiency with a hyperbolic tangent function $\tanh \lambda\left(f-f_{0}\right)$, where the two parameters $\lambda$ and $f_{0}$ are determined by fitting to the detection efficiency points. We obtained the following values: $\lambda=4.0 \times 10^{4} \mathrm{MeV}^{-1} \mathrm{~cm}^{2}$ s and $f_{0}=8.41 \times 10^{-7} \mathrm{MeV} \mathrm{cm}^{-2} \mathrm{~s}^{-1}$ with a $\chi^{2}$ of 0.93 with 9 degrees of freedom. We obtained an efficiency $>80 \%$ with energy flux $>3 \times 10^{-5} \mathrm{MeV} \mathrm{cm}^{2} \mathrm{~s}^{-1}$ and $\sim 100 \%$ for energy flux $>5 \times 10^{-5}$ $\mathrm{MeV} \mathrm{cm}^{-2} \mathrm{~s}^{-1}$.

\subsection{The 1FLT Catalog construction}

The final 1FLT catalog is composed of 142 unique transient sources not associated with any 4FGL-DR2 emitters: 64 sources detected in the nominal 120 months and 78 in the shifted 120 months. Seventy-two 1FLT sources with $25<\mathrm{TS}<30$ are flagged as low-confidence (as reported in Sec. 2.1). The source designation is 1FLT JHHMM+DDMM.

Out of these 142 1FLT sources, 108 1FLT sources were detected only once, i.e. in one single TBIN, while 34 other distinct 1FLT sources displayed significant gamma-ray emission in more then one TBIN. These 34 sources listed in 1FLT correspond to their most significant detection in each relative cluster of multiple flaring episodes. In case of multiple detections of positionally consistent candidates, the source name is based on the position of the candidate detection with the largest value of TS.

The 1FLT method allowed us to collect also 60 detections associated with the Sun and 27 associated with 14 of the brightest Fermi-LAT $\gamma$-ray bursts (GRBs). 


\section{Source association}

To identify candidate counterparts of the 1FLT sources we used two approaches: the Bayesian method, extensively described in the xFGL catalogs (see, e.g. [9]), and the positional method, which relies solely on the location of the counterpart inside the error ellipse. We performed the Bayesian association procedure on each of the 240 skies for all the candidate seeds with a localization evaluation, each month (i.e., TBIN) being an independent sky. Similarly to what was done in the xFGL catalogs, in the 1FLT we retained associations if they had a posterior probability of at least $80 \%$.

For 1FLT sources without a Bayesian association, we searched the $95 \%$ confidence error ellipse for the presence of promising, not yet known, $\gamma$-ray emitting sources. This search was carried out using the SSDC Tools ${ }^{3}$ and VOU-Blazar Tool ${ }^{4}$. This method enables the association of the sources with radio surveys that are not used by the Bayesian association method.

For 1FLT sources with large positional error ellipses more than one candidate counterpart was found. In these cases, as well as when no candidate counterparts were found, we flagged the 1FLT source as unassociated.

The census of the 1FLT sources is reported in Table 1. 1FLT includes 6 sources that are associated with a source from previous FGL catalogs (1-3FGL) but that do not have a counterpart in the 4FGL-DR2 list. We find also correspondence in position and time for 4 sources of the 2FAV catalog [10] whose analysis is on a one-week time interval.

\begin{tabular}{llccc}
\hline \hline Class & Class Description & 1FLT & High Confidence & Low Confidence \\
\hline BCU & Blazars of Uncertain Type & 70 & 41 & 29 \\
FSRQ & Flat Spectrum Radio Quasar & 24 & 14 & 10 \\
BLL & BL Lacertae object & 1 & & 1 \\
OTHER & Non Blazar object & 7 & 2 & 5 \\
UNASS & Unassociated & 40 & 13 & 27 \\
\hline
\end{tabular}

Table 1: Census of 1FLT Sources

\section{Properties of 1FLT sources}

The 1FLT distribution in Figure 1(a) extends to softer $\Gamma$ values (median value of $\Gamma \sim 2.7$ ) than that of the 4LAC sources (median value of $\Gamma \sim 2.2$ or 2.5 if we consider only 4LAC FSRQs). Twenty-six 1FLT sources exhibit softer spectral indices than the softest 4LAC FSRQ. The identification of these soft-spectrum sources shows that integrating over monthly timescales allowed us to investigate the soft part of the gamma-ray source distribution, which is usually suppressed (or confused with the low energy component of the $\gamma$-ray background) when analyzed over longer, multi-year timescales.

For all of the 1FLT sources associated with blazars we collected the available multifrequency data in order to infer the synchrotron peak frequency $v_{\text {peak }}^{\mathrm{S}}$ of the observed broadband SED which

3https://tools.ssdc.asi.it/

4http: //wWw . openuniverse.asi.it/ 


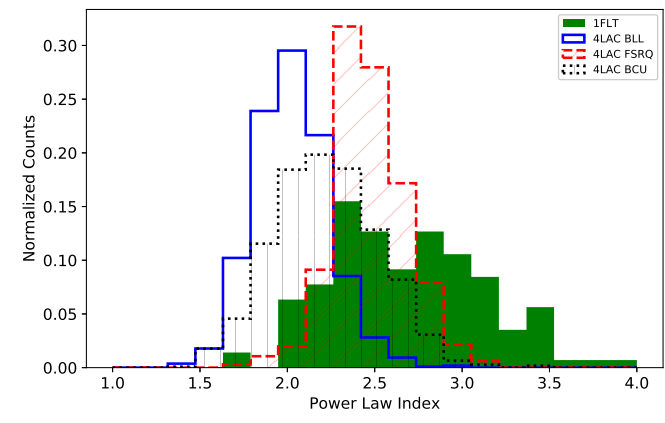

(a)

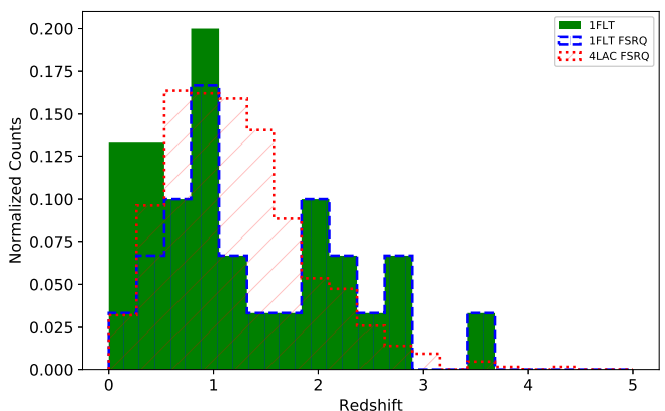

(b)

Figure 1: (a) Normalized distribution of power-law index for 1FLT sources (green filled histogram) and 4LAC sources. The blue line represents the 4LAC BL Lac objects, the red dashed line 4LAC FSRQ and the black dotted line 4LAC BCU objects. (b) Redshift distributions for 1FLT (green shaded region), 1FLT FSRQ (blue dashed line) and 4LAC FSRQ (red dotted line) sources.

was in turn used to perform a SED-based classification. In this procedure we used a compilation of broad-band archival data. The estimation of $v_{\text {peak }}^{\mathrm{S}}$ relies on a 3rd-degree polynomial fit of the low-energy hump of the SED which is performed on a source-by-source basis (the procedure was described in [11]). With this procedure we reconstructed the $v_{\text {peak }}^{\mathrm{S}}$ for only 651 FLT sources due to the limited multifrequency sampling. We detected only low-synchrotron-peaked sources $\left(v_{\text {peak }}^{\mathrm{S}}<10^{14} \mathrm{~Hz}\right)$ with soft spectra.

Redshifts are available for a total of 30 1FLT sources: all FSRQs, 1 BCU, 1 BLL, 3 RG and 1 non-blazar object. The 1FLT redshift distribution in Figure 1(b) shows a peak at low redshift $(<1)$. This peak is due to the contribution of 1FLT misaligned AGN. These sources show a larger jet inclination angle, where de-boosted radiation makes the relativistic jet radiation weaker and more difficult to detect in $\gamma$ rays [12]. About 50\% of FSRQs are detected at $z>1$. Another effect is that there is an additional peak at $\mathrm{z}>2$, unlike the $4 \mathrm{LAC}$ distribution which includes only a small fraction of objects at $\mathrm{z}>2$. This shows that the 1FLT method is well suited for detecting high-redshift blazars.

For each 1FLT source that has a counterpart with a known redshift, we computed the $\gamma$-ray luminosity using the $\Lambda \mathrm{CDM}$ cosmological parameters obtained by Planck [13]; in particular, we used $h=0.67, \Omega_{m}=0.32$, and $\Omega_{\Lambda}=0.68$, where the Hubble constant $H_{0}=100 h \mathrm{~km} \mathrm{~s}^{-1} \mathrm{Mpc}^{-1}$. The 1FLT sources show greater $\gamma$-ray luminosities, possibly because they are detected during flaring states, and softer $\gamma$-ray spectra than the sources detected by integrating over years-long periods. When integrating over short time intervals, sporadic flaring activity is detected from a sub-luminous population whose integrated emission would not have been detected over longer time intervals, due to their weak quiescent flux being merged into the integrated background gamma-ray emission. These sources are very bright during a flare, but show a faint long-term integrated $\gamma$-ray flux, which limits their possibility to be detected significantly on the long-term periods typically considered for xFGL catalogs. 


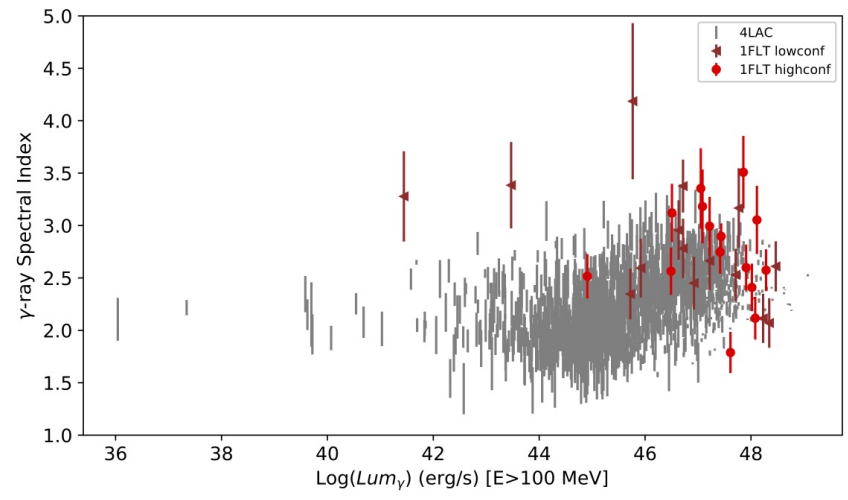

Figure 2: Power-law index of all 1FLT sources, plotted as a function of the $\gamma$-ray luminosity (100 MeV $300 \mathrm{GeV}$ ). Red filled circles represent 1FLT high-confidence sources; brown filled triangles represent 1FLT low-confidence sources. In gray are shown 4LAC data points for comparison.

\section{Incremental FLT}

Starting from this work, we have constructed a pipeline dedicated to the routine search of the transient sources on monthly time scale, complementary to the routine search on shorter time scale (day and week) done by the Fermi-LAT Flare Advocate activity and Fermi All-sky Variability Analysis (FAVA). The incremental source list (iFLT) will be updated yearly at link www.ssdc.asi.it/fermi_iflt. The current iFLT version is constructed on two years of data. These data start coherently with the end of the 1FLT data both for the nominal and the shifted period of time. The pipeline collected $108 \gamma$-ray detections on 48 months (nominal TBINs from 120 to 143 and shifted ones from 120.5 to 143.5). In this number there are 9 detections of the Sun and 55 new sources with respect to 4FGL-DR2. Among these, 25 sources can been associated to a source of the 4FGL Data Release 3 (12 years of data, 4FGL-DR3, [14]). The number of new $\gamma$-ray emitters is 30 . This sample is composed by 7 BCUs, 8 FSRQs, 1 SSRQ, 2 Gigahertz Peaked Spectrum (GPS) quasars and 12 unassociated sources. To identify candidate counterparts we use only the positional method. The median value of the iFLT sources power-law index is 2.7 , as for the 1FLT sources. One source is detected in more than one TBIN. Twenty sources are flagged as low-confidence.

Of particular interest is the detection of the bright transient Nova Reticuli 2020 (MGAB-V207), reported by several multiwavelength ATels from July 2020. The iFLT detected this source in TBIN 143 and 143.5 (July - August 2020) respectively with TS $=507$ and TS $=30$. This detection shows the potentiality of this work in the present and future multi-wavelength campaigns. Furthermore this iFLT target is positionally consistent with the 4FGL-DR3 source J0358.5-5432 associated to PMN J0358-5434. This shows that different time binning permits us to distinguish emitters of different type.

The sky locations of the 1FLT and iFLT sources together with Sun, GRB and 4FGL-DR3 detections are shown in Figure 3. 


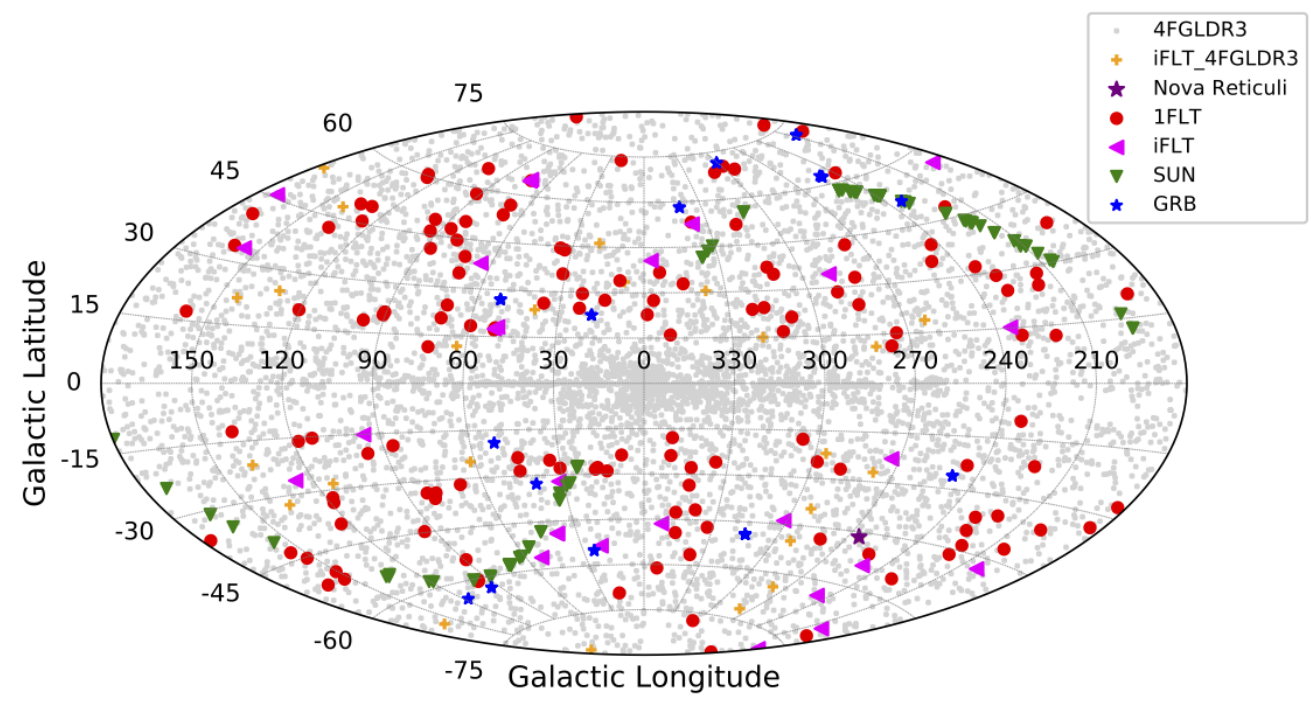

Figure 3: Aitoff projection of 1FLT sources represented in Galactic coordinates (red filled circles), iFLT sources (magenta filled triangles), Sun detections (green filled triangles) and GRB detections (blue filled stars) superimposed on 4FGL-DR3 sources represented in gray. The purple star is the Nova Reticuli detection and the orange crosses represent iFLT detections associated to 4FGL-DR3.

\section{Summary and Conclusions}

In this work we have investigated the Fermi-LAT sky on monthly timescales to search for sources which are not detected when integrating on timescales of years and/or that are not already hosted in the Fermi-LAT xFGL general catalogs. We found that:

- The 1FLT catalog and the iFLT list are mainly populated by soft sources with a median value of power-law index of $\sim 2.7$ compared to $\sim 2.3$ for the 4FGL-DR2 (the same for the 4FGL-DR3). This confirms that these soft sources are not distinguishable from the diffuse $\gamma$-ray background when considered over multi-year integration times.

- This study found that there are more FSRQs found when using a monthly binning compared to catalogs using data averaged over longer intervals. This is due both to BL Lacs that are in general less variable around $1 \mathrm{GeV}$ and to FSRQs whose strong gamma-ray activity is mainly seen only during flaring events. If the flare intensity is fainter than the integrated background over years, we lose the capability to detect these FSRQs in long-time integrated catalogs.

- In the 1FLT (and in the iFLT) we detected sources only when they were in an active flaring phase. For this reason they show a high $\gamma$-ray luminosity with respect to the 4LAC sources whose fluxes were calculated over a longer integration time.

The 1FLT catalog comprises $142 \gamma$-ray sources of which $\sim 70 \%$ are associated with soft AGN-type counterparts, principally BCU and FSRQ. Approximately $28 \%$ of 1 FLT sources remain 
unassociated. This fraction is similar to the percentage of unassociated sources found in the FermiLAT general catalogs. The 72 1FLT sources that have TS between 25 and 30 and are detected in only one of the monthly time bins should be regarded as low confidence, given their $~ 34 \%$ probability of being statistical fluctuations. Out of the 142 monthly detections we found only 4 correspondences with the $2 \mathrm{FAV}$ catalog whose analysis is on a one-week time interval. This shows that integrating over different time intervals does not reproduce the same $\gamma$-ray sky and that the discovery space of Fermi-LAT remains large. The 1FLT catalog is available online for an easy and fast visualization at www.ssdc.asi.it/fermi $1 \mathrm{flt}$.

A pipeline dedicated to the routine search of the transient sources on monthly time scale has been constructed starting from this work. The iFLT list will be updated yearly.

\section{Acknowledgments}

The Fermi-LAT Collaboration acknowledges support for LAT development, operation and data analysis from NASA and DOE (United States), CEA/Irfu and IN2P3/CNRS (France), ASI and INFN (Italy), MEXT, KEK, and JAXA (Japan), and the K.A. Wallenberg Foundation, the Swedish Research Council and the National Space Board (Sweden). Science analysis support in the operations phase from INAF (Italy) and CNES (France) is also gratefully acknowledged. This work performed in part under DOE Contract DE-AC02-76SF00515.

\section{References}

[1] W. B. Atwood et al., ApJ 697 (2009) 1071.

[2] J. Ballet et al., 2005.11208.

[3] A. A. Abdo et al., ApJ 722 (2010) 520.

[4] F. Damiani et al., ApJ 483 (1997) 370.

[5] S. Ciprini et al., in AIP Conference Proceedings, vol. 921, pp. 546-547, AIP, 2007, DOI.

[6] M. Wood et al., PoS (ICRC2017) 824 (2017) .

[7] J. R. Mattox et al., ApJ 461 (1996) 396.

[8] G. Principe et al., $A \& A 618$ (2018) A22.

[9] S. Abdollahi et al., ApJS 247 (2020) 33.

[10] S. Abdollahi et al., ApJ 846 (2017) 34.

[11] M. Ackermann et al., ApJ 810 (2015) 14.

[12] A. A. Abdo et al., ApJ 720 (2010) 912.

[13] Planck Collaboration, A\&A 571 (2014) A16 [1303. 5076].

[14] B. Lott et al, ICRC2021 (2021). 